\title{
Uncertainty Analysis of Camera Parameters Computed with a 3D Pattern
}

\author{
Carlos Ricolfe-Viala and Antonio-José Sánchez-Salmerón \\ Department of Systems Engineering and Automatic Control \\ Polytechnic, University of Valencia,Valencia, Spain \\ \{cricolfe, asanchez@isa.upv.es\}, http://www.isa.upv.es
}

\begin{abstract}
Camera calibration is a necessary step in 3D modeling in order to extract metric information from images. Computed camera parameters are used in a lot of computer vision applications which involves geometric computation. These applications use camera parameters to estimate the $3 \mathrm{D}$ position of a feature in the image. Depending on the accuracy of the computed camera parameter, the precision of the position of the image feature in the 3D scene vary. Moreover if previously the accuracy of camera parameters is known, one technique or another can be choose in order to improve the position of the feature in the 3D scene.

Calibration process consists of a closed form solution followed by a non linear refinement. This non linear refinement gives always the best solution for a given data. For sure this solution is false since input data is corrupted with noise. Then it is more interesting to obtain an interval in which camera parameters are contained more than an accurate solution which is always false.

The aim of this paper is to present a method to compute the interval in which the camera parameter is included. Computation of this interval is based on the residual error of the optimization technique. It is know that calibration process consists of minimize an index. With the residual error of the index minimization an interval can be computed in which camera parameter is. This interval can be used as a measurement of accuracy of the calibration process.
\end{abstract}

Keywords: camera calibration, accuracy evaluation, interval estimation, 3D pattern.

\section{Introduction}

Calibration of cameras is considered as an important issue in computer vision. It allows extracting metric information from 2D images. Accurate calibration of cameras is especially crucial for applications that involve quantitative measurements such as dimensional measurements. Calibration process computes camera parameters which are divided into internal and external. Internal camera parameters determine how the image coordinates of a point is derived, given the spatial position of the point with respect to he camera. Internal parameters are also those which define the quality of the image. They measure the camera distortions. These parameters are non linear related with the data input coordinates. By the other hand, position of the camera with 
respect to the scene is defined with the external parameters. In this case a linear relation exists.

Existing camera calibration techniques can be divided into several categories. From the point of view of the calibration object they use, they can be classified into four categories. First uses 3D reference calibration object. In this case, calibration object consist of two or three planes orthogonally to each other. This requires an elaborated calibration apparatus but calibration can be done very efficiently [2]. Second, calibration process is based of the observation of a planar pattern shown at different orientations [11]. Any knowledge about camera motion is necessary. The calibration setup is easier but less accurate results are computed. Third 1D calibration object is used. In this case very easy calibration setup is necessary [12]. However accuracy of the computed parameters is worst. The fourth group is called selfcalibration. These calibration techniques are those which do not use any calibration object because only image point correspondences are required. Just by moving a camera in a static scene, the rigidity of the scene provides in general two constraints [7] on the cameras' internal parameters. If the images are taken by the same camera with fixed internal parameters, correspondences between three images are sufficient to recover both internal and external parameters.

All calibration techniques give a closed form solution for the camera parameters. Afterwards, an iterative scheme is used improve the results and to estimate parameters with non linear relation. They are radial distortion and geometrical misalignments. Taking into account that a non linear iterative scheme is done, final camera parameters are similar if one calibration object or another is used. They just give an initial value of the parameters to initialize non linear estimation and the iterative scheme give "the best" solution. Moreover, the computed parameter will never be the right ones since the input data is computed with noise. They will be the best which fit given data. From this point of view it is more interesting to obtain an interval in which the parameter is contained than computed an optimal solution for given data which will be wrong for sure.

In this paper a method to compute a interval in which a camera parameter is contained is presented. It is based on the residual error coming from the minimization algorithm. Depending on the minimization algorithm used to calibrate the camera the interval will be bigger or smaller. In this case the process is particularized to the calibration process using 3D calibration object since it gives more accurate solution using a closed form solution [1],[8]. One advantage of this calibration method is that any inverse matrix is computed. That means, from a mathematical point of view that, this method is robust and good performance is achieved with no well conditioned matrixes. First a brief presentation of the calibration process is done. Second, computing interval method is presented following with the results of both simulated and real experiments.

\section{Camera Calibration Using a 3D Pattern}

If $X_{i}=\left(x_{i}, y_{i}, z_{i}, 1\right)^{T}$ represents a point of the 3D calibration object and $U_{i}=\left(u_{i}, v_{i}, 1\right)^{T}$ is its position in the $2 \mathrm{D}$ image plane expressed in homogeneous coordinates, they are related with the following expression: 


$$
s U_{i}=C[R t] X_{i} \quad \text { with } \quad C=\left[\begin{array}{ccc}
\alpha & 0 & u_{0} \\
0 & \beta & v_{0} \\
0 & 0 & 1
\end{array}\right]
$$

where $s$ is a arbitrary scale factor, $[R t]$ are the extrinsic parameters. These are the rotation and translation which relates the camera coordinate system with the scene coordinate system. $C$ contains the intrinsic parameters where $\left(u_{0}, v_{0}\right)$ corresponds to the coordinates of the principal point, $\alpha$ and $\beta$ are the scale factors of the image in $u$ and $v$ axes. Calibration process consist of compute both internal and external camera parameters. To compute them intermediate ones are defined. In this case they correspond to the elements of the projection matrix. This matrix is called $M$ and it is defined as $M=C[R t]$. This involves that $s U_{i}=M X_{i}$. To obtain the projection matrix elements starting from a set of points' coordinates $X_{i}$ and $U_{i}$, they are arranged in a matrix in the following way:

$$
\left[\begin{array}{cccccccccccc}
x_{1} & y_{1} & z_{1} & 1 & 0 & 0 & 0 & 0 & -u_{1} \cdot x_{1} & -u_{1} \cdot y_{1} & -u_{1} \cdot z_{1} & -u_{1} \\
0 & 0 & 0 & 0 & x_{1} & y_{1} & z_{1} & 1 & -v_{1} \cdot x_{1} & -v_{1} \cdot y_{1} & -v_{1} \cdot z_{1} & -v_{1} \\
. . & . . & . . & . . & . . & . . & . . & . . & . . & . . & . . & . . \\
x_{n} & y_{n} & z_{n} & 1 & 0 & 0 & 0 & 0 & -u_{n} \cdot x_{n} & -u_{n} \cdot y_{n} & -u_{n} \cdot z_{n} & -u_{n} \\
0 & 0 & 0 & 0 & x_{n} & y_{n} & z_{n} & 1 & -v_{n} \cdot x_{n} & -v_{n} \cdot y_{n} & -v_{n} \cdot z_{n} & -v_{n}
\end{array}\right] \cdot\left[\begin{array}{c}
m_{11} \\
m_{12} \\
. . \\
m_{33} \\
m_{34}
\end{array}\right]=0
$$

it can be called:

$$
A \cdot m=0
$$

where $m$ is the column vector with the unknown elements of the projection matrix and $n$ the number of points used in the calibration process. The projection matrix elements are the elements of the eigen vector connected with the smallest eigen value of the matrix $A^{T} A[8]$.

Since the noise in the points' coordinate measurements, the solution is always false. In order to obtain more information from the calibration process an interval in which the parameter is included, is computed. One way to do it is explained in the following section.

\section{Mean and Variance of Projection Matrix Elements}

Mean and the variance of projection matrix elements depend on the calibration algorithm and the noise level of the points' coordinates and also their situation in the world. It is supposed that they are situated in such a way that the resulting matrix $A^{T} A$ is well conditioned. The aim is to compute a covariance matrix $\Lambda_{m}$ of dimensions (12x12) of the estimated projection matrix. In this section a method to compute them is presented assuming that the calibration process is carried out computing the eigen vectors of the matrix $A^{T} A$. Computation of the covariance matrix is based on the following theorem [3] [10]. Given a symmetric matrix $B$ of dimensions $n x n$, exists an orthogonal matrix $H$ which accomplished the following expression:

$$
H^{-1} B H=\operatorname{diag}\left\{\lambda_{1}, \lambda_{2}, \ldots . \lambda_{n}\right\}
$$


where $\operatorname{diag}\left\{\lambda_{1}, \lambda_{2}, \ldots \ldots \lambda_{n}\right\}$ is a diagonal matrix with the eigen values of $B$ starting from the small one.

$$
\lambda_{1}<\lambda_{2} \leq \ldots \ldots \leq \lambda_{n}
$$

Each column of the matrix $H$ is called eigen vector and it is associated with an eigen value $\lambda_{1}, \lambda_{2}, \ldots . . \lambda_{n}$.

$$
H=\left[\begin{array}{llll}
h_{1} & h_{2} & \ldots & h_{n}
\end{array}\right]
$$

Elements of $B$ corrupted with noise are called $B_{e}$ and then $B_{e}=B+\Delta_{B}$. The perturbation of the eigen values using the perturbation of the matrix $B$ is defined with

$$
\Delta_{\lambda n}=h_{n}^{T} \Delta_{B} h_{n}
$$

the perturbation of the associated eigen vector is

$$
\Delta_{h n}=H \Delta H^{-1} \Delta_{B} h_{n}
$$

where $\Delta$, for the first eigen vector is

$$
\Delta=\operatorname{diag}\left\{0,\left(\lambda_{1}-\lambda_{2}\right)^{-1}, \ldots \ldots,\left(\lambda_{1}-\lambda_{n}\right)^{-1}\right\}
$$

This theorem allows computing the eigen vector perturbation given the perturbation of the initial matrix $\Delta_{B}$. If the projection matrix $m$ is estimated computing the eigen values of $A^{T} A$, this theorem allows to compute the perturbation of the estimated elements of the projection matrix. Vector $m$ corresponds to the eigen value $\lambda_{I}$. In order to compute this perturbation, last equation is used. The eigen vector which contains the elements of the matrix projection $m$ is the first one since it is associated with the small eigen value. The perturbation of the estimated vector $m$ is

$$
\Delta_{m}=H \Delta H^{-1} \Delta_{A^{T} A} m
$$

If this equation is rearranged in order to put the perturbation of the matrix $A^{T} A$ in the right side, in a column vector the following expression arises:

$$
\Delta_{m}=H \Delta H^{-1} D \Delta_{A^{T} A}
$$

where $D$ are the elements of the vector $m$ rearranged since the perturbation of $A^{\prime} A$ is now in a column vector $\Delta_{A}{ }^{T}$.

$$
D=\left[\begin{array}{lllll}
m_{1} I_{12} & m_{2} I_{12} & m_{3} I_{12} & \ldots . & m_{12} I_{12}
\end{array}\right]
$$

$I$ represents a unit (12x12) matrix. From now to the end of the paper $\Delta_{A}{ }^{T}$ and $\Delta_{A}$ represent the perturbation of the matrix $A^{T} A$ and $A$ but arranged as a column vector. In order to express $\Delta_{m}$ as a function of the perturbation of the vector $\Delta_{A}$, it is necessary to express $\Delta_{A}{ }_{A}^{T}$ as a linear function of $\Delta_{A}$. If $\Delta_{A}{ }^{T}{ }_{A}=f\left(\Delta_{A}\right)=K \Delta_{A}$ then

$$
\Delta_{m}=H \Delta H^{-1} D K \Delta_{A}
$$

If $L=H \Delta H^{-1} D K$ then $\Delta_{m}=L \Delta_{A}$ and therefore the variance of the computed elements of the projection matrix will be 


$$
\Lambda_{m}=L^{T} \Delta_{A}{ }^{T} \Delta_{A} L=L^{T} \Lambda_{A} L
$$

These are the result of applying the theorem to the calibration process and rearrange the matrices to express $\Lambda_{m}$ as a function of $\Lambda_{A}$. In order to use this theorem, several terms should be defined before. These are matrix $K$, the value of $\Delta_{A}$ and the covariance matrix $\Lambda_{A}$. They are defined in the following sections.

\subsection{Interval Computation Method}

Interval computation method can be summarized as follows. Using (1), (2) the relation between $\Delta_{m}$ and $\Delta_{A}$ is defined as

$$
\Delta_{m}=H \Delta H^{-1} D(F+G) \Delta_{A}=L \Delta_{A}
$$

where

$$
\begin{gathered}
\Delta=\operatorname{diag}\left\{0,\left(\lambda_{1}-\lambda_{2}\right)^{-1}, \ldots . .\left(\lambda_{1}-\lambda_{n}\right)^{-1}\right\} \\
F=\left[\begin{array}{lllll}
F_{1} & F_{2} & F_{3} & \ldots . & F_{2 n}
\end{array}\right] \\
G=\left[\begin{array}{lllll}
G_{1} & G_{2} & G_{3} & \ldots . & G_{2 n}
\end{array}\right] \\
F_{i}=\left[\begin{array}{llllll}
a_{i 1} I_{12} & a_{i 2} I_{12} & a_{i 3} I_{12} & \ldots . & a_{i 12} I_{12}
\end{array}\right]^{T} \\
G_{i}=\left[\begin{array}{ccccc}
a^{T} O_{12 \times 11} & O_{12 \times 12} & O_{12 \times 12} & \ldots . & O_{12 \times 12} \\
O_{12 \times 12} & O_{12 \times 1} a^{T} O_{12 \times 10} & O_{12 \times 12} & \ldots . & O_{12 \times 12} \\
O_{12 \times 12} & O_{12 \times 12} & O_{12 \times 2} a^{T} O_{12 \times 9} & \ldots . & O_{12 \times 12} \\
\ldots . & \ldots . & \ldots . & \ldots . & \ldots . \\
O_{12 \times 12} & O_{12 \times 12} & O_{12 \times 12} & \ldots . & O_{12 \times 11} a^{T}
\end{array}\right]
\end{gathered}
$$

Therefore the variance of the elements of the projection matrix is defined as

$$
\Lambda_{m}=L^{T} \Delta_{A}{ }^{T} \Delta_{A} L=L^{T} \Lambda_{A} L
$$

where $\Lambda_{\mathrm{A}}$ is computed with

$$
\Lambda_{A}=\left[\begin{array}{ccccc}
\Lambda_{A 1} & O_{24 \times 24} & O_{24 \times 24} & \ldots & O_{24 \times 24} \\
O_{24 \times 24} & \Lambda_{A 2} & O_{24 \times 24} & \ldots & O_{24 \times 24} \\
O_{24 \times 24} & O_{24 \times 24} & \Lambda_{A 3} & \ldots & O_{24 \times 24} \\
\ldots & \ldots & \ldots & \ldots & \ldots \\
O_{24 \times 24} & O_{24 \times 24} & O_{24 \times 24} & O_{24 \times 24} & \Lambda_{A n}
\end{array}\right]
$$

Once the variance of the projection matrix elements is computed, it is possible to propagate this variance to the camera parameters. If a camera parameter $p$ is related with the matrix projection elements with a function $p=g(m)$, the variance of the parameter $p$ is

$$
\Lambda_{p}=\nabla g(\bar{m}) \Lambda_{m} \nabla g(\bar{m})^{T}
$$


where $\nabla g(\bar{m})$ represents the partial derivative of the function $g$ for the computed matrix projection. Taking into account that the perturbation is modeled with a Gaussian distribution, the interval in which the parameter is contained can be computed using $95 \%$ of confidence degree. This is computed multiplying the standard deviation by 2 .

\section{Experimental Results}

In order to test how well the variance estimation works several simulations have been carried out and also interval with real data from images has been calculated.

\subsection{Simulations with Synthetic Data}

A set of points are generated in two planes, and a camera is situated in the scene as is shown in figure 1 . The camera is situated $1 \mathrm{~m}$ away form the $\mathrm{Y}$ axis, with an angle of incidence of the camera optical axe of 45 degrees with the X-Y plane. The features of the virtual camera are $U_{0}=300 V_{0}=200 \alpha=1580 \beta=1580$.

The camera is in the scene and a chessboard of $20 \times 20$ points is generated. The images of the points in the scene are using the projection matrix formed with the ideal camera parameters. Afterwards a noise level is added to the scene points coordinates and to the image points' coordinates. With the corrupted coordinates, the camera calibration is done. An estimated value and an interval in which it is included, is computed. This interval is obtained with the $95 \%$ of the variance. Since the real value is known, it can be compared with the estimated value and the computed interval. The most representative results have been obtained changing the noise level in the points' coordinates, and the number of points used in the camera calibration process. Figure 2 shows the results for the internal parameter. Remaining camera parameters have similar behavior. The left side figure shows how the estimated interval changes with the noise level in the coordinates increase. In this case 50 points are used. Since the noise level increase, the residual error is bigger and the estimated interval increase also. The central figure shows the effects of the number of points used as input data in the calibration process. The noise level is constant to 1 pixel in the image coordinates and 1 millimeter in the scene points' coordinates. If the number of points increases the computed solution is less deviated from the real one and therefore the residual error of the minimization decrease. As a consequence the interval in which the camera parameter is contained decrease also. The right side figure compares the computed interval with 10 points and 1 pixel and 1 millimeter noise level, with several camera calibrations using a Montecarlo simulation. Estimations are represented with one dot. Border straight lines shows mean of the intervals computed with each calibration. This figure shows that the interval computed with the previous analysis is correct because border lines include $95 \%$ of the estimated values for the internal parameter $U_{0}$. Therefore, the value of the camera parameters will be always in this interval. This information will be very useful in order to mix information from several methods. The behavior of remaining parameters is very similar to this one. 


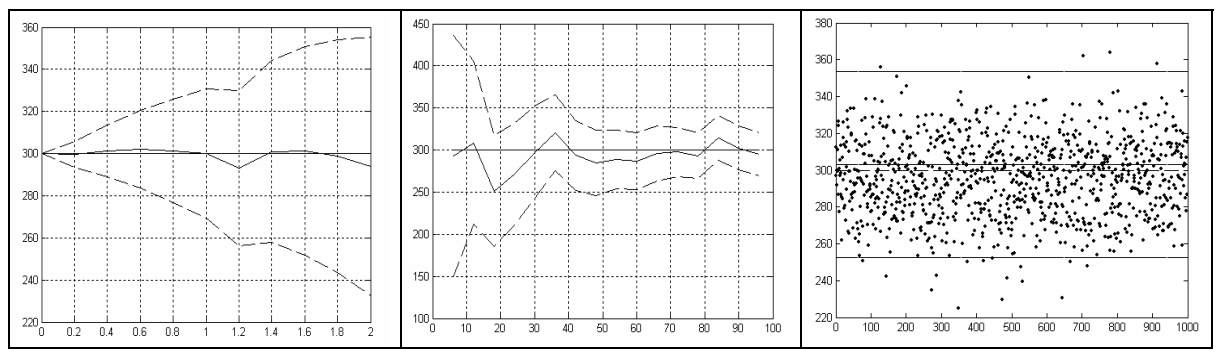

Fig. 1. Interval estimation of $U_{0}$. Straight line is the real value, continuous line is the estimated value and dotted line is the interval. Left - the noise level changes and the number of points is fixed. Center - the points number changes and the noise level is fixed. Right - the noise level and number of points is fixed and several camera calibrations have been performed. Each dot corresponds to one estimation. Border straight lines corresponds to the mean of interval computed with the presented method.

\subsection{Experiments with Real Data}

In this case images of a real calibration object has been taken. They are shown in figure 2. Camera is situated half a meter away from the origin of coordinates of the scene. About 150 points are used in each calibration process. Calibration is carried out and using the residual error of the minimization algorithm, the interval in which the camera parameters are contained is computed. Table attached to figure 2 shows the results of the calibration process with real data. The camera has been calibrated 100 times to test if the computed interval includes the true value. Right column shows the mean results together with its standard deviation and the left one the computed parameters and the computed uncertainty for one calibration process. Really, the computed interval includes the true value of the parameter.

\begin{tabular}{|c|c|c|c|}
\hline & & One Calibration & Mean Values \\
\hline & $\boldsymbol{\sigma}^{2}$ & 1.124 & 1.027 \\
\hline $\boldsymbol{U}_{\boldsymbol{0}}$ & $329.248 \pm 12.429$ & $317.259 \pm 1.648$ \\
\hline $\boldsymbol{V}_{\boldsymbol{\theta}}$ & $232.268 \pm 15.521$ & $245.157 \pm 0.574$ \\
\hline $\boldsymbol{\alpha}$ & $1257.654 \pm 24.268$ & $1279.681 \pm 1.876$ \\
\hline $\boldsymbol{\beta}$ & $1125.681 \pm 29.2658$ & $1139 \pm 1.658$ \\
\hline $\boldsymbol{T} \boldsymbol{x}$ & $4.258 \pm 5.689$ & $-0.158 \pm 0.268$ \\
\hline & $\boldsymbol{T} \boldsymbol{y}$ & $79.267 \pm 10.598$ & $75.684 \pm 4.589$ \\
\hline $\boldsymbol{T} \boldsymbol{z}$ & $68.268 \pm 8.547$ & $73.895 \pm 3.258$ \\
\hline
\end{tabular}

Fig. 2. 3D pattern and estimated camera parameters and its intervals

\section{Conclusions}

A method to determine the variance of the projection matrix elements based on computing the eigen vector of the matrix $A^{\prime} A$ has been presented. Using the residual error of the minimization process the standard deviation of the projection matrix 
elements can be computed. From the point of view of $3 \mathrm{D}$ reconstruction process, interval in which the camera parameter is included is more useful than an accurate solution for a given set of data which is always false. Interval can be use to chose one or another 3D reconstruction method depending on the quality of the camera parameters and it can be computed with the presented method.

\section{Acknowledgement}

This work was financially supported by the Spanish government (CICYT project number DPI2004-08353-C03-02), European Community FEDER funds and European Community research funds (Project number 500095-2).

\section{References}

[1] J. R. Cooperstock, Requirements for camera calibration: must accuracy come with high price? IEEE Work shop on applications of compueter vision. 2004

[2] O. Faugeras, Three dimensional computer vision: A geometric viewpoint. Cambridge, 1993.

[3] G.H.Golub, C.F.Van Loan, Matrix computation, third edition. The Jonh Hopkins University Press 1996

[4] R. I. Hartley, "In defence of the eight point algorithm". IEEE Transactions on pattern analysis and machine intelligence. 1997

[5] R.Hartley, A. Zisserman Multiple view geometry in computer vision. Cambridge 2000

[6] K. Kanatani, Statistical Optimization for geometric computation, Springer, June 1995.

[7] S.J. Maybank, O.D. Faugeras. "A theory of self-calibration of a moving camera". The international Journal of computer vision. 1992

[8] J. Salvi, X. Armangué, J. Battle A comparative review of camera calibrating methods with accuracy evaluation. Pattern Recognition 35, 2002

[9] J. Weng, P. Cohen, M. Herniou, "Camera calibration with distortion models and accuracy evaluation" IEEE transactions on pattern analysis and machine intelligence. 1992

[10] J. Weng, T.S. Huang, N. Ahuja, "Motion and structure from two perspective views: algorithms, error analysis and error estimation" IEEE transactions on pattern analysis and machine intelligence. 1989

[11] Z. Zhang, "A flexible new technique for camera calibration". IEEE transactions on pattern analysis and machine intelligence 2000

[12] Z. Zhang, "Calibration with one-dimensional objects". Microsoft technical report. 2002 